\title{
Uma abordagem de Ecossistemas de Software para o domínio de e-Learning
}

\section{Alternative Title: A Software Ecosystem approach to e-Learning domain}

\author{
Welington Veiga \\ Programa de Pós-graduação em \\ Ciência da Computação \\ Universidade Federal de Juiz de Fora \\ welington.veiga@ice.ufjf.br
}

\author{
Fernanda Campos \\ Programa de Pós-graduação em \\ Ciência da Computação \\ Universidade Federal de Juiz de Fora \\ fernanda.campos@ufff.edu.br \\ José Maria David \\ Programa de Pós-graduação em \\ Ciência da Computação \\ Universidade Federal de Juiz de Fora \\ jose.david@ufjf.edu.br
}

\author{
Regina Braga \\ Programa de Pós-graduação em \\ Ciência da Computação \\ Universidade Federal de Juiz de Fora
}

regina.braga@ice.ufjf.br

\begin{abstract}
RESUMO
O domínio de e-learning é caracterizado pela fragmentação de soluções e múltiplas implementações similares. Nesse artigo é apresentada uma abordagem para permitir o desenvolvimento, compartilhamento e reuso de serviços educacionais por meio da perspectiva de Ecossistemas de Software. Através da extensão dos atuais sistemas de informação dos ambientes de e-learning, conhecidos como Ambientes Virtuais de Aprendizagem, busca-se criar uma plataforma de um ecossistema que permita colaboração inter-organizacional. A proposta apresentada foi avaliada através um estudo de caso, verificando os conceitos, a arquitetura e as tecnologias utilizadas.
\end{abstract}

\section{Palavras-Chave}

Ecossistemas de e-Learning, Ecossistemas de Software.

\begin{abstract}
The e-learning domain is characterized by fragmented solutions and multiple similar implementations. This paper presents an approach to enable the development, sharing and reuse of educational services through the Software Ecosystem perspective, through the extension of existing systems information of elearning environments, known as Virtual Learning environments. To become platforms of an ecosystem that enables interorganizational collaboration. The proposal was evaluated by a case study, to verify its feasibility, as well as the concepts, architecture and technologies used.
\end{abstract}

\section{Categories and Subject Descriptors}

D.2.13 [Reusable Software]: Domain engineering, Reusable libraries, Reuse models.

\footnotetext{
Permission to make digital or hard copies of all or part of this work for personal or classroom use is granted without fee provided that copies are not made or distributed for profit or commercial advantage and that copies bear this notice and the full citation on the first page. To copy otherwise, or republish, to post on servers or to redistribute to lists, requires prior specific permission and/or a fee.

SBSI 2016, May 17-20, 2016, Florianópolis, Santa Catarina, Brazil. Copyright SBC 2016.
}

\author{
General Terms \\ Management, Design, Human Factors, Standardization.
}

\section{Keywords}

E-learning Ecosystems, Software Ecosystems.

\section{INTRODUÇÃO}

No domínio de e-Learning a necessidade de reuso é um assunto cujo interesse de pesquisa é recorrente. Em especial, em relação à recursos educacionais [26][13], através dos quais o conceito de unidades educacionais independentes e de pequena granularidade, que possam ser utilizadas para a composição de programas que atendam às necessidades educacionais específicas, estão presentes desde a década de 90 [25]. Essas pesquisas enfatizam o uso de repositórios de Objetos de Aprendizagem (OAs) reutilizáveis, de definições de metadados e de ontologias para busca de conteúdos educacionais. Além do reuso de OAs, diferentes serviços educacionais como chats, fóruns, processadores de textos, jogos educacionais e vídeo conferências podem auxiliar o processo de ensino e aprendizagem e não estão diretamente relacionados ao escopo de uma plataforma educacional [16].

Apesar desses esforços de pesquisa, existe uma grande fragmentação no domínio e dificuldades na integração de serviços, recursos e ferramentas aos Ambientes Virtuais de Aprendizagem (AVAs). Os AVAs consistem em sistemas de informação para a distribuição de conteúdo educacional online e facilitação de toda a experiência de ensino-aprendizagem em torno desse conteúdo [30]. Os AVAs estão presentes nos ambientes de e-learning tanto no Ensino Superior [27] quanto nas empresas [4].

Estas dificuldades foram observadas pelos autores a partir de estudos exploratórios realizados no mercado de treinamento corporativo no contexto de e-learning, bem como na experiência com cursos de Educação à Distância (EAD) oferecidos no contexto acadêmico. Nestes estudos foram realizadas coletas de informações com profissionais envolvidos em atividades de $e$ learning. Além disso, foi realizada uma revisão bibliográfica a 
respeito do problema identificado. Dessa forma foram obtidas evidências da fragmentação de soluções de software no domínio de e-learning, bem como o grande número de plataformas diferentes [15][11], que é problema abordado no presente trabalho.

As dificuldades de integração, compartilhamento e reuso no domínio de e-learning ocorrem em um contexto onde a conectividade e interdependência entre empresas é um assunto de crescente interesse [3]. Essas empresas não funcionam mais como unidades independentes, tornando-se dependentes de componentes e infraestruturas fornecidos por terceiros [19]. Nesse cenário, as inovações não são mais frutos de uma única empresa, e sim co-inovações construídas a partir da interdependência entre diferentes empresas [2].

Como forma de compreender essas relações complexas, a perspectiva de Ecossistemas de Software (ECOS) pode ser utilizada para analisar a indústria de software, sob um ponto de vista inspirado em ecossistemas naturais e de negócios, onde se considera não só o software em si, mas sua dependência de componentes e infraestrutura de terceiros, usuários e a interação entre cada um dos envolvidos [3][19].

Este trabalho apresenta uma proposta de adoção da abordagem de Ecossistema de Software no domínio de e-learning a partir da incorporação de características de uma plataforma de ecossistemas com o objetivo de apoiar o desenvolvimento, compartilhamento, reuso de serviços educacionais em um contexto interorganizacional.

Além desta introdução, este trabalho está assim organizado: a seção 2 aborda a metodologia empregada, a seção 3 discute a fundamentação teórica utilizada, a seção 4 apresenta os trabalhos relacionados, a seção 5 descreve a proposta apresentada neste trabalho descrevendo suas características e arquitetura conceitual. A seção 6 apresenta a avaliação realizada e a seção 7 as considerações finais, limitações e trabalhos futuros.

\section{METODOLOGIA}

Um projeto de pesquisa científica possui sete características fundamentais [14], que consistem em: (I) criação de um artefato para (II) atender a um problema específico, (III) cuja utilidade deve ser explicitada através de uma avaliação apropriada e (IV) os resultados e contribuições da pesquisa compartilhado com profissionais interessados e academia. Para assegurar sua validade, $(\mathrm{V})$ as investigações devem ser conduzidas com rigor e (VI) as possíveis formas de solução analisadas e por fim (VII) os resultados devem ser comunicados.

Esta pesquisa atende aos sete critérios ao propor (I) uma abordagem (II) favorecer o desenvolvimento, compartilhamento e reuso de serviços educacionais em um contexto interorganizacional, (III) avaliá-lo por meio de um estudo de caso e (IV) apresentar os resultados à comunidade acadêmica e aos profissionais através do presente artigo e de recursos disponibilizados na internet. Mantendo (V) o rigor metodológico durante seu desenvolvimento, (VI) analisando as soluções existentes no contexto de Ecossistemas de e-Learning e (VII) garantindo a publicação dos resultados.

\section{FUNDAMENTAÇÃO TEÓRICA}

$\mathrm{O}$ uso de conceitos de Ecossistemas Digitais no domínio de $e$ learning não é recente. Em 2002 Cowley [8] já utilizavam o termo ecossistema para definir papeis e requisitos para a construção de um ambiente de e-Learning, dividindo-os entre bióticos (estudantes, instrutores) e abióticos (conteúdo, tecnologia). No mesmo ano, Wilkson [32] identificava características de ecossistemas ao definir elementos e relações entre eles, como requisitos para um ambiente de e-learning, propondo um modelo de Ecossistema de e-Learning com foco em aspectos relacionados aos reuso de OAs, na taxonomia de recursos, nas características do domínio de e-learning, no processo de aprendizagem e em sua dimensão tecnológica.

Uma visão mais abrangente de Ecossistemas de e-Learning é apresentada por Brodo [6], que define Ecossistema de e-Learning como "o termo utilizado para descrever todos os componentes requeridos para implementar um ambiente de e-Learning", esses componentes incluem a noção de fatores bióticos e abióticos, e são divididos em três grupos: (A) os provedores de conteúdo, que proveem o conteúdo educacional; (B) os consultores, que ajudam a desenvolver, definir os processos educacionais e oferecer suporte às tecnológias e (C) infraestrutura, que inclui todos os recursos necessários para o gerenciamento, entrega $\mathrm{e}$ acompanhamento do e-learning .

O modelo teórico proposto por Chang e Gutl [7] é particularmente interessante por sua abordagem holística, baseando-se na definição ecológica de ecossistema como a encontrada na Enciclopédia Britânica: "complexo de organismos vivos, seu ambiente físico, e todas as suas inter-relações em uma unidade de espaço" para identificar no domínio de e-learning fatores bióticos, abióticos e todas as suas inter-relações dentro do ambiente, que é delimitado por fronteiras bem definidas (Figura 1).

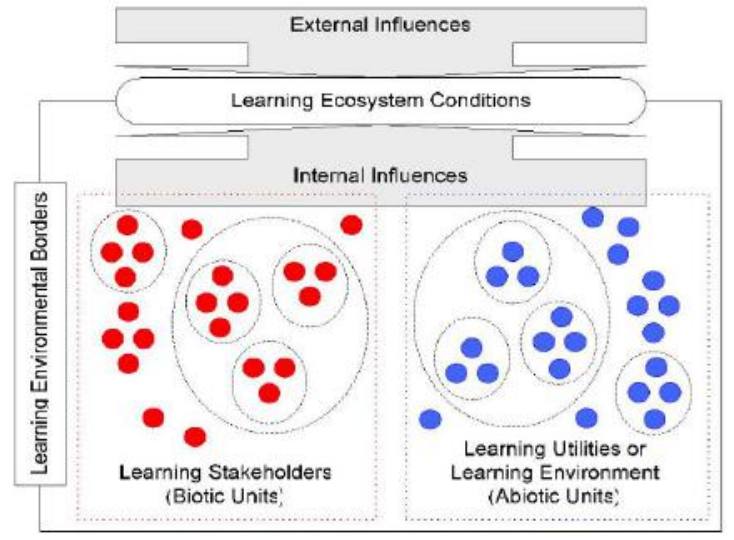

Figura 1 - Ecossistema de e-Learning [8].

Essa definição incorpora indivíduos, comunidades educacionais e atores (professores, tutores, provedores de conteúdo, designers instrucionais, pedagogos), artefatos e recursos (conteúdo e aspectos pedagógicos de conteúdo multimídia, tecnologia, ferramentas), e interações dentro de determinada fronteira do ecossistema definida por pressões internas e externas (objetivos educacionais, evolução do conhecimento, aspectos culturais e sociológicos).

Nesses Ecossistemas de e-Learning, embora incluam elementos de ECOS, não foi identificada uma fundamentação teórica comum que relacione esses dois tipos de Ecossistemas Digitais. Além disso, foi verificado que existem pontos importantes amplamente discutidos e aprofundados no contexto de ECOS que não fazem parte da literatura relacionada a Ecossistemas de e-Learning, tais como evolução, variabilidade, 
ferramentas, frameworks e comunidades de desenvolvedores externos. Petterson [26] utiliza conceitos de ECOS para analisar o domínio de e-learning, mas não propõe uma forma de solucionar o problema da fragmentação e múltiplas implementações sob essa perspectiva.

Devido à natureza sócio-técnica dos ECOS, é difícil determinar de forma genérica suas características. No entanto, algumas características chave com foco em processos técnicos do desenvolvimento e evolução dos ECOS são identificadas por Lettner et al. [22], com base em uma revisão de literatura. E, embora não sejam características determinantes para definir um ECOS, são parâmetros relevantes para sua análise: (C1) existência de desenvolvedores internos e externos, estendendo as fronteiras de uma organização (BOSCH, 2012); (C2) existência de uma plataforma tecnológica comum, que pode ser uma base tecnológica, um framework extensível e adaptável, um conjunto componentes ou de soluções prontas e adaptáveis; (C3) existência de um controle central, que implica em um ciclo de vida da plataforma independente das aplicações externas compatíveis com a mesma; (C4) possibilidade de contribuições externas em sua plataforma, podendo ser na forma de código aberto com possibilidade de incorporação de código externo, interfaces ou pontos de extensão; (C5) arquitetura com suporte à variabilidade, onde a plataforma suporta adaptações para as especificidades de cada cliente, permitindo que aplicações sejam construídas por desenvolvedores externos baseadas nas configurações específicas de funcionalidades, aplicações e componentes oferecidos pela plataforma; (C6) ativos compartilhados, uma característica herdada da abordagem de linha de produto de software, que é uma estratégia para reuso intra-organizacional são os ativos compartilhados, recursos reutilizáveis compartilhados para a criação de aplicações compatíveis, enquanto os ECOS são uma abordagem para reuso inter-organizacional. [19]; (C7) derivação automática de produtos ou derivação suportada por ferramentas, uma vez que há suporte à variabilidade e criação de novas funcionalidades com base em configurações específicas, há a possibilidade de criação de novos produtos apenas com essa configuração por parte de desenvolvedores externos e até mesmo usuários de forma automática ou suportada por ferramentas; (C8) soluções específicas de clientes incorporadas à plataforma, onde uma solução para um cliente específico tem potencial para atender a outros clientes da plataforma; (C9) ferramentas, frameworks e padrões, para auxiliar o desenvolvimento, evolução e manutenção da própria plataforma, dos recursos compartilhados entre diferentes clientes e demais extensões e (C10) canal de distribuição, é um recurso apresentado por alguns ECOS onde há um canal de distribuição de software desenvolvido por terceiros acessível aos clientes da plataforma, frequentemente mantido pela organização responsável pela plataforma.

A partir das características de ECOS, é possível analisar os Ecossistemas de e-Learning sob essa perspectiva e orientar a proposição de utilização da abordagem de ECOS para o domínio educacional.

\section{TRABALHOS RELACIONADOS}

São relacionados os trabalhos que abordam a integração de ferramentas externas e serviços a plataformas educacionais sob uma perspectiva de ecossistemas. Embora existam vários trabalhos que proponham o uso de Serviços Web e SOA em conjunto com Ecossistemas de e-Learning [17][24], esses trabalhos não cobrem aspectos de ECOS importantes, como o tipo de API oferecida para desenvolvedores externos, o tipo de recurso acessível para serviços externos e a abertura da proposta para contribuições externas. Nesta seção são apresentados os trabalhos que consideram essas características.

O Jampots [12] é uma implementação de Ecossistema de eLearning baseado em mashups, combinação de informações de várias fontes em diferentes formatos oferecidos através de APIs de terceiros gerando novas funcionalidades, e constitui-se de uma plataforma para o projeto colaborativo, disponibilização, compartilhamento, gerenciamento e recriação de conteúdo educacional para usuários finais. A arquitetura do Jampots é composta por quatro camadas, que são: camada de conteúdo de eLearning, camada de sindicalização de conteúdo de e-Learning, camada de componentes mashups e camada de aplicações mashups. No entanto, embora haja possibilidade de criação de funcionalidades por parte de usuários, há uma limitação em relação a dependências de APIs heterogêneas de terceiros para a composição desses serviços.

O Dippler [20] é a plataforma de um ecossistema que não se limita à estrutura, arquitetura e implementação, mas considera uma perspectiva mais ampla, sócio tecnológica, envolvendo aspectos políticos, econômicos, acadêmicos e tecnológicos. O Dippler é um Ecossistema de e-Learning baseado em alguns requisitos importantes do ponto de vista de ECOS, como código fonte aberto, modularidade, permite o desenvolvimento baseado em comunidades, integração com padrões e especificações do domínio de e-Learning, interoperabilidade com blogs, wikis, repositórios de conteúdo educacional e possibilidade de adição de novos serviços educacionais e personalização de sua aparência, onde os estudantes usam seus blogs pessoais para compartilhar recursos educacionais como SlideShare ${ }^{1}$ e YouTube $^{2}$ Várias mídias sociais podem ser incorporadas ao ecossistema, a partir de RSS ou iFrame widgets. Além disso, o Dippler suporta o reuso de OAs criados e hospedados pelas ferramentas, tais como: Questr, desenvolvida pelos autores para autoria de OA, ferramenta de autoria LeMill ${ }^{3}$ e o repositório de OA Waramu ${ }^{4}$.

A Experience API (xAPI), é uma especificação que torna possível a coleta de dados sobre uma ampla gama de experiências de aprendizagem de uma pessoa, tanto online quanto off-line. Permite que diferentes sistemas compartilhem informações educacionais utilizando um vocabulário compartilhado. A definição da xAPI foi iniciada pela ADL, porém é aberta, seu desenvolvimento é dirigido pela comunidade, e permite livre implementação [1]. Devido a definição de uma interface e um formato de armazenamento de dados comuns, a xAPI estabelece uma especificação que suporta a definição de um Ecossistema de e-Learning [18]. Os dados são armazenados em repositórios chamados de Learning Record Store (LRS), que oferecem uma API para o armazenamento de informações de experiências de aprendizagem, e ferramentas externas atuam como "produtores" ou "consumidores" dessas informações [1].

\footnotetext{
${ }^{1}$ http://www.slideshare.net/

${ }^{2}$ https://www.youtube.com/

${ }^{3}$ http://lemill.net/

${ }^{4}$ http://trac.htk.tlu.ee/waramu
} 
A infraestrutura Ludos tem como objetivo a inclusão de elementos de gamificação em Ecossistema de e-Learning existentes [29], considerando os seus fatores bióticos (professores, instrutores, estudantes, pedagogos, especialistas), abióticos (multimídia, software, tecnologia, equipamentos, $\mathrm{AO}$ e plataforma) e sua fronteira (evolução do conhecimento, objetivos educacionais e aspectos culturais). Além disso, novos fatores bióticos (produtores e desenvolvedores e serviços educacionais com elementos de gamificação) e abióticos (Repositório de Conquistas, OAs com elementos de gamificação, Ferramenta de Autoria e Serviços Educacionais com gamificação) nesse ecossistema. A arquitetura da infraestrutura visa permitir a geração e armazenamento de informações de gamificação (como pontos, medalhas e títulos conquistados) e consulta aos mesmos, através da captura de dados de serviços e OAs e disponibilização desses dados através de uma API.

Analisados sob a perspectiva de ECOS (Tabela-1), os trabalhos apresentados não cobrem aspectos como a possibilidade de contribuição externa à plataforma, suporte de ferramentas e bibliotecas que favoreçam a construção de serviços compatíveis, atendem parcialmente aspectos como o uso de ativos compartilhados e a possibilidade de incorporar recursos de terceiros à plataforma. Dessa forma existem oportunidades de aprofundamento em relação a características de ECOS no domínio de e-learning para favorecer o desenvolvimento, compartilhamento e reuso de soluções educacionais em um contexto inter-organizacional.

Tabela 1. Comparação de características de ECOS entre trabalhos de Ecossistemas de e-Learning

\begin{tabular}{|c|c|c|c|c|}
\hline Característica & Jampots & Dippler & xAPI & LUDOS \\
\hline C1 & Sim & Sim & Sim & Sim \\
\hline C2 & Sim & Sim & Não & Sim \\
\hline C3 & Sim & Sim & Não & Não \\
\hline C4 & Não & Não & Não & Não \\
\hline C5 & Sim & Sim & Sim & Sim \\
\hline C6 & Sim & Não & Não & Não \\
\hline C7 & Não & Não & Não & Não \\
\hline C8 & Sim & Não & Não & Sim \\
\hline C9 & Não & Não & Sim & Sim* \\
\hline C10 & Sim & Não & Não & Não \\
\hline
\end{tabular}

\section{PROPOSTA DE SOLUÇÃO}

Este trabalho aborda o uso da perspectiva de ECOS no contexto de e-learning, formado pela interação entre diferentes atores, comunidades, organizações e serviços educacionais a partir de uma plataforma comum.

Nesse contexto, os AVAs podem ser considerados "plataformas" dos ambientes de e-learning. No entanto, já existe uma diversidade desses ambientes [11], e propor um novo iria de encontro aos objetivos de favorecer o reuso. Além disso, dificulta a adoção da solução proposta, uma vez que as organizações já possuem seus AVAs. Portanto, é um requisito permitir uma abordagem baseada em ecossistema com ênfase em reuso a partir dos AVAs atuais.
Dessa forma faz parte dessa proposta uma infraestrutura que permita a transformação de AVAs existentes em plataformas de um ECOS do domínio de e-learning. Nesta seção são discutidas as características da abordagem proposta, sua arquitetura conceitual e desenvolvimento.

\subsection{Características}

A partir da análise comparativa dos trabalhos relacionados e das necessidades identificadas para atingir ao objetivo definido, foram definidas as características que devem ser adicionadas aos AVAs que compõem requisitos funcionais e não funcionais da abordagem proposta:

Integração de serviços externos: deve ser possível adicionar ao ecossistema serviços com diferentes funcionalidades e objetivos através de uma API, sem que seja necessário alterar a plataforma, facilitando a integração de serviços de outras organizações.

Estabelecimento de um modelo comum para o domínio de elearning: para que haja troca de informações entre os serviços e a plataforma é necessário que exista um domínio comum compreendendo as principais entidades do domínio de e-Learning, como participante (estudante, professor, monitor), curso, turma, grupos, notas, onde a plataforma é sempre a autoridade em relação às informações do domínio.

Controle de autorização: para acesso à leitura e gravação de informações, a infraestrutura deve oferecer uma forma de controle de autorização de baixa granularidade para acessos de serviços a recursos oferecidos na plataforma. Essa característica permite que diferentes serviços possuam diferentes níveis de integração com a plataforma, de acordo com sua aplicação, objetivos e nível de confiança no fornecedor.

Uso de metadados educacionais: o uso de metadados educacionais nos serviços do ecossistema é importante para que facilitadores e especialistas possam avaliar se determinado serviço é adequado de acordo com as necessidades e objetivos pedagógicos de determinado contexto.

Poucas restrições na criação de serviços compatíveis: os serviços devem possuir poucas restrições, seja em relação a aspectos tecnológicos, implementações de segurança ou comunicação.

\subsection{Arquitetura de Software de Referência}

Para oferecer um padrão de solução como referência de arquitetura que atenda aos requisitos propostos ao mesmo em que mantem a flexibilidade para soluções específicas para um problema específico em determinado domínio, são definidas Arquiteturas de Software de Referência (ASR) [28][9].

A arquitetura proposta é uma ASR orientada a serviços web, e independente de tecnologia e plataforma. Toda comunicação entre os componentes é feita através de serviços RESTful com dados em formato JSON. É um formato utilizado em diferentes domínios, incluindo ecossistemas [21][23], e suportado por bibliotecas, frameworks e ferramentas em todas as linguagens de programação e plataformas populares (Figura 2).

O conjunto de serviços web disponíveis para a comunicação entre os serviços educacionais e a plataforma formam a API de integração, que define os recursos, a estrutura das requisições e as possíveis respostas. Uma das principais características da API é ter um modelo de permissões com fina granularidade, garantindo 
flexibilidade no nível de integração entre cada serviço e a plataforma, através de escopos implementados a partir do protocolo OAuth $2.0^{5}$.

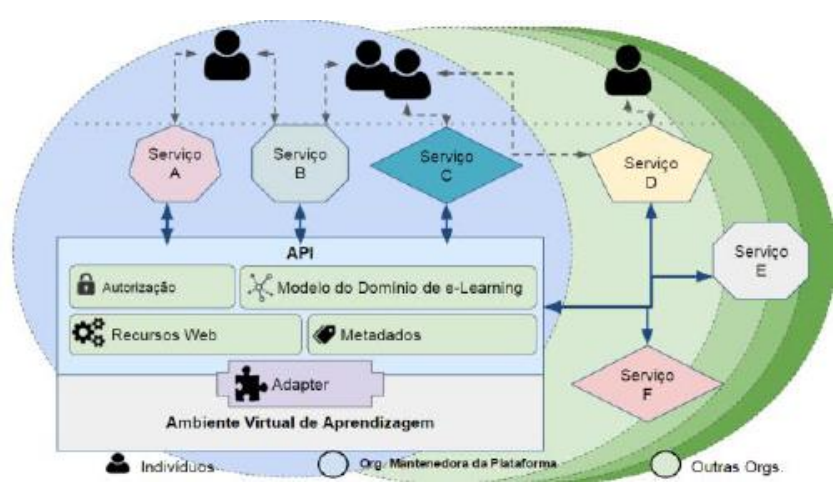

Figura 2 - Arquitetura Conceitual.

Os serviços disponíveis na API são baseados no modelo do domínio de e-learning definido por meio de uma ontologia (Figura 3). A partir dessa ontologia as principais entidades envolvidas na integração podem ser relacionadas, e a definição dos escopos associados a cada entidade permite que sejam visualizados pelo professor os recursos da API que determinado serviço pode consumir. Os escopos têm a forma (<entidade>.<tipo de operação>), como participant.read ou courseclass.write, para ler dados de um participante ou escrever dados em turmas, por exemplo.

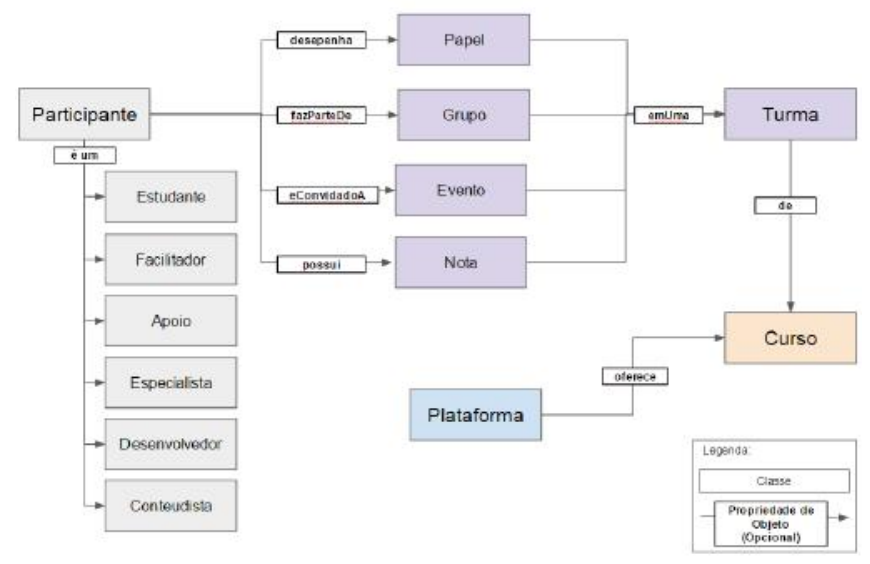

Figura 3 - Classes e propriedades de objetos da ontologia de domínio.

A conexão entre a infraestrutura e o AVA é feita através de um Adapter, devendo ser uma fina camada de software na forma de plugin, extensão ou alteração no próprio AVA. O Adapter é responsável por traduzir as chamadas à API, que são independentes do AVA, para as peculiaridades e características de cada um deles e, para isso, deve ser capaz de alinhar o modelo de dados utilizado pela API para o modelo de domínio utilizado internamente pelo AVA (Figura 4).

Para a criação de serviços educacionais compatíveis com a infraestrutura, basta manter a compatibilidade com a API que exige que apenas três recursos web sejam suportados pelo serviço

\footnotetext{
${ }^{5}$ http://oauth.net/2/
}

(para atender ao protocolo de segurança e disponibilizar metadados) e utilizar apenas os recursos do lado da plataforma que desejar, ignorando os demais. Os serviços podem ser desenvolvidos em qualquer linguagem e plataforma.

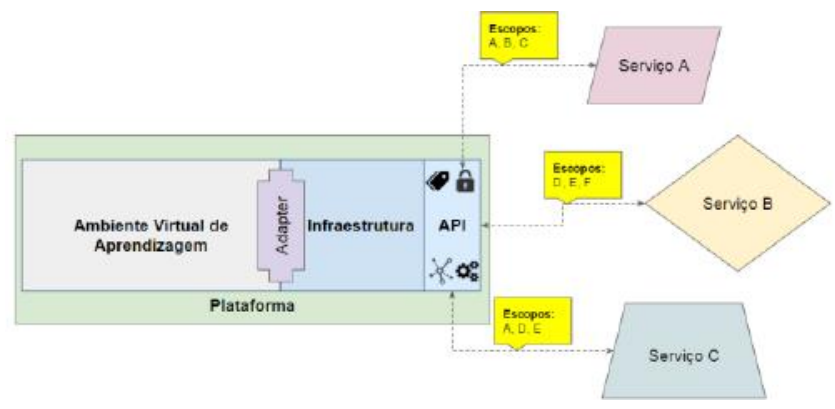

Figura 4 - Comunicação entre serviços e a plataforma

\subsection{Alguns aspectos da implementação}

Uma primeira versão da abordagem apresentada foi desenvolvida com o objetivo de demonstrar a viabilidade técnica da proposta bem como permitir a obtenção de evidências de verificação dos objetivos definidos.

Dessa forma, um fator importante é a integração a AVAs existentes. Foram escolhidos dois AVAs com características distintas para aplicar a abordagem proposta e mostrar sua generalidade. O primeiro AVA escolhido foi o Moodle, um AVA de código livre desenvolvido em PHP e utilizando tecnologias abertas com amplo suporte a plug-ins de terceiros. O segundo escolhido (AVA II) é proprietário de código fechado, e desenvolvido utilizando tecnologias proprietárias. Suas funcionalidades são desenvolvidas exclusivamente pela organização responsável pelo mesmo, com uma base de usuários superior a $400 \mathrm{mil}$, de diferentes organizações. Igualmente importante é o desenvolvimento de um serviço educacional compatível com proposta. Assim, foi desenvolvido o Taughtology, um serviço para ensino de lógica com elementos de gamificação e interação entre os estudantes. Nesta seção são apresentados detalhes do desenvolvimento desses recursos.

O Moodle é um AVA de código livre desenvolvido em PHP com suporte a diferentes SGBDs, utilizado por milhões de organizações no mundo, incluindo empresas e universidades, com amplo suporte a plugins. Utilizando a documentação oficial disponível no website do Moodle e utilizando como material adicional o código fonte de plug-ins existentes, foi desenvolvido um plugin que inclui uma implementação da infraestrutura proposta na linguagem de programação PHP desenvolvida no contexto e um Adapter que abstrai as chamadas à recursos do domínio de e-learning em chamadas específicas aos recursos do Moodle, além de disponibilizar interfaces de usuário dentro do AVA para adição e acesso de serviços como atividades nos cursos oferecidos através da plataforma (Figura 5).

O AVA II possui usuários ativos distribuídas entre diferentes organizações clientes, acessando a mesma instância do mesmo, em um modelo multi tenant. $\mathrm{O}$ acesso ao código fonte e às características técnicas do AVA II foi disponibilizado para esta pesquisa pela empresa responsável pelo seu desenvolvimento. Esta empresa é especializada em treinamento corporativo e possui plataformas educacionais e tecnologias próprias. Sob o ponto de vista técnico, o AVA II foi desenvolvido na linguagem Java em uma plataforma J2EE distribuída, e os recursos e customizações 
são desenvolvidos internamente pela própria organização. $\mathrm{O}$ suporte a extensão é feito através de páginas customizadas, que podem conter código específico para determinadas organizações. A partir do acesso à documentação interna e entrevista com desenvolvedores da equipe de desenvolvimento deste produto, foi desenvolvida uma página customizada que atua como Adapter para integração com uma implementação da infraestrutura utilizando as linguagens Clojure e Java (Figura 6).

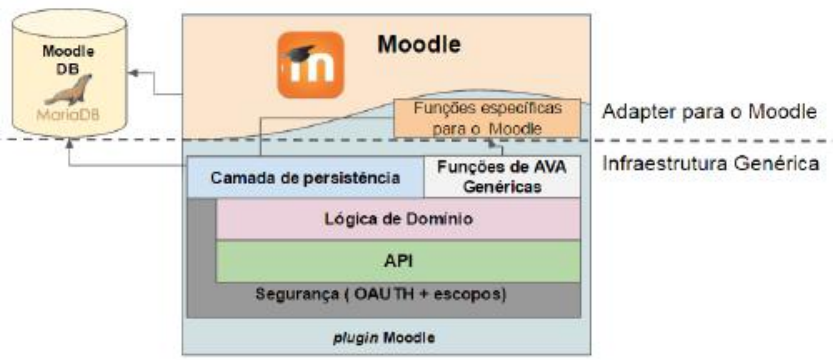

Figura 5 - Arquitetura do plugin Moodle .

O serviço educacional Taughtology (taught + tautology) foi desenvolvido para auxiliar no ensino de lógica utilizando elementos de gamificação, como níveis (fácil, médio, difícil e insano). Pontos foram atribuídos a cada resposta correta, que aumentam de valor de acordo com o nível do problema solucionado. Os problemas são chamados de desafios. Além disso, um estudante pode desafiar um colega a acertar um desafio que ele tenha errado, de forma que os dois ganham pontos caso este acerte. Não faz parte do escopo desta seção discutir os aspectos pedagógicos envolvidos no projeto deste Serviço Educacional.

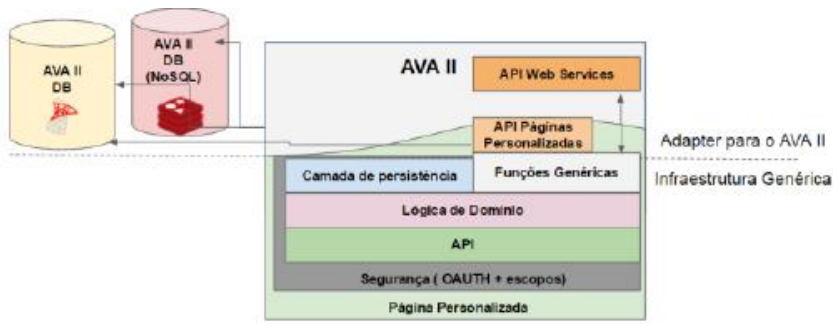

Figura 6 - Página Personalizada para o AVA II.

Sob o ponto de vista técnico, em seu desenvolvimento foi utilizada uma arquitetura em camadas, utilizando a linguagem de programação Java na versão 8 em conjunto com o framework Spring Boot (Figura 7).

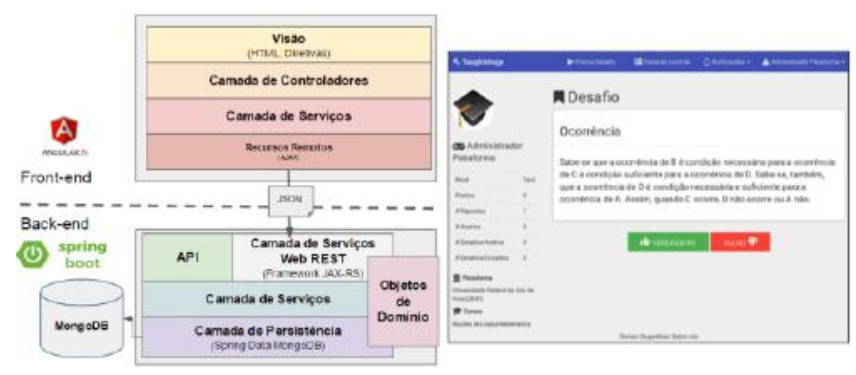

Figura 7 - Arquitetura e interface do Taughtology.

Os escopos exigidos pelo Taughtology são: $\mathrm{O}$ acesso à lista de participantes da turma atual, (coursesclass.participants.read), aos dados usuário autenticado (participant.read), e do curso atual (course.read). Para realizar a integração à plataforma, o professor deve permitir que o serviço tenha acesso a esses recursos.

\section{AVALIAÇÃO DA SOLUÇÃO}

Para a avaliação, é necessário considerar a abordagem proposta segundo o ponto de vista dos atores envolvidos em seu contexto de aplicação. Segundo Dresch [14] o estudo de caso é uma pesquisa empírica que busca melhor compreender um fenômeno contemporâneo, normalmente complexo no seu contexto real. São considerados valiosos, pois, permitem descrições detalhadas dos fenômenos normalmente baseados em fontes de dados diversas e asseguram que a investigação e o entendimento do problema sejam feitos em profundidade, como desvantagem os resultados obtidos dessa forma dificilmente podem ser generalizados [31].

Foi planejado um cenário de utilização considerando os atores envolvidos no processo e a infraestrutura necessária para sua execução. Para a realização dos estudos de caso, foram definidas as seguintes fases, adaptadas de Wholin [31]: (I) definição do estudo de caso; (II) formulação do objetivo; (III) planejamento; (IV) execução e observação das evidências; e (V) a apresentação das evidências observadas.

\subsection{Formulação do objetivo}

O objetivo do estudo de caso foi definido e formulado de acordo com a abordagem Goal/Question/Metric (GQM) [31], enunciado por: "Analisar a abordagem de ECOS para o domínio educacional, com a finalidade de demonstrar a viabilidade da proposta, dos conceitos e das tecnologias envolvidas com respeito à integração, compartilhamento e reuso de serviços externos a AVAs sob o ponto de vista de atores do ecossistema no contexto e-Learning".

\subsection{Planejamento}

O planejamento do estudo de caso inclui para cada uma de suas etapas (Tabela 02): o ator responsável, a descrição, os passos que devem ser realizados e os pontos a serem observados

Tabela 2. Planejamento do estudo de caso.

\begin{tabular}{|c|c|}
\hline 1 Etapa & Adição do Adapter ao AVA \\
\hline Ator & Administrador \\
\hline Descrição & $\begin{array}{l}\text { Adição do suporte à abordagem através do Adapter (plugin } \\
\text { no moodle e página customizada no AVA II) }\end{array}$ \\
\hline Passos & Adicionar Adapter ao AVA \\
\hline $\begin{array}{c}\text { Pontos de } \\
\text { Observacão }\end{array}$ & Detalhes técnicos que devem ser considerados. \\
\hline 2 Etapa & Integração de Serviço Externo a Curso \\
\hline Ator & Professor \\
\hline Descrição & Integração de serviço à plataforma. \\
\hline Passos & $\begin{array}{l}\text { 1. Acessar um curso em que lecione. } \\
\text { 2. Adicionar um serviço externo } \\
\text { 3. Dar as permissões (escopos) que considera } \\
\text { adequados ao serviço. } \\
\text { 4. Disponibilizar o serviço no curso. }\end{array}$ \\
\hline $\begin{array}{l}\text { Pontos de } \\
\text { Observação }\end{array}$ & $\begin{array}{l}\text { Detalhes técnicos que devem ser considerados. } \\
\text { Complexidade na identificação das permissões necessárias. } \\
\text { Dificuldade em identificar se o serviço é ou não adequado a } \\
\text { partir dos metadados disponibilizados. }\end{array}$ \\
\hline 3 Etapa & Acesso ao Serviço Externo \\
\hline Ator & Estudante \\
\hline Descrição & Acesso a um serviço integrado a partir do AVA. \\
\hline Passos & $\begin{array}{l}\text { 1. Acessa o curso do qual é participante. } \\
\text { 2. Acessa ao serviço externo a partir da } \\
\text { plataforma. }\end{array}$ \\
\hline $\begin{array}{c}\text { Pontos de } \\
\text { Observação }\end{array}$ & Detalhes técnicos que devem ser consider \\
\hline
\end{tabular}




\subsection{Execução e coleta de evidências}

Uma vez disponibilizado o ambiente, as etapas foram realizadas observadas sob o ponto de vista dos atores. Na Etapa I o usuário Administrador acessa o ambiente e entra na página de plugins do Moodle, faz o upload do plugin desenvolvido a partir de um arquivo compactado, instalando-o como qualquer outro plugin para a plataforma. Nesse processo nenhum detalhe técnico é informado. No AVA II, a página customizada é enviada para um serviço de storage através de uma área administrativa onde é necessário conhecer detalhes técnicos, no entanto é um procedimento conhecido pelos administradores desta plataforma.

Na Etapa II o usuário Professor I acessa o Moodle e navega até a sua página de cursos, selecionando um de seus cursos, o Introdução à Lógica. Na página de edição do curso, adiciona uma atividade e na tela seguinte seleciona o tipo de atividade "Serviço Externo", sendo redirecionado para a página de configuração da atividade criada. Na página de configuração da atividade o Professor informa a URI do Taughtology, e imediatamente os escopos solicitados pelo serviço para integração são exibidos, assim como os metadados. No AVA II esse procedimento ocorre de forma análoga, o Professor I acessa a página de customizada, seleciona o curso Introdução à Lógica Matemática, e executa o mesmo procedimento acima (Figura 8).

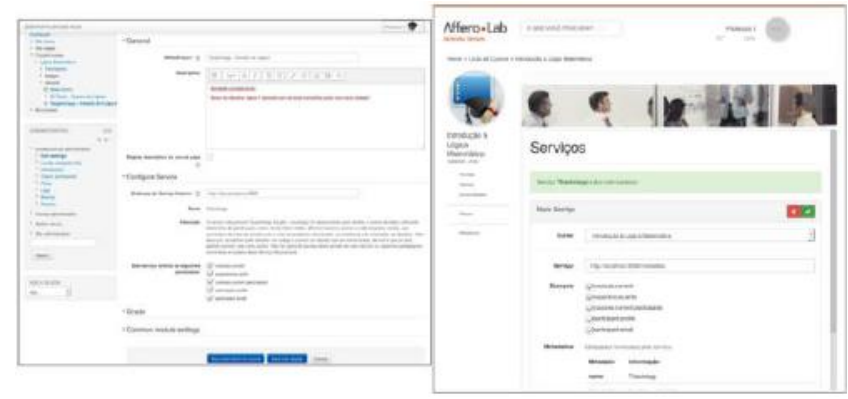

Figura 8 - Etapa II no Moodle (Esq.) e no AVA II (Dir.).

Na Etapa III o Estudante I acessa o ambiente Moodle e navega até o curso Introdução à Lógica. Na lista de atividades visualiza o Taughtology, com o título e descrição informados pelo Professor. Ao clicar na atividade é redirecionado para uma página dentro da plataforma onde pode utilizar o serviço, que exibe na interface seu nome, o nome curso em que está sendo executado, bem como o nome da instituição responsável pela plataforma. Ao clicar no botão "abrir em outra janela", o serviço fica disponível de forma independente do Moodle, em uma segunda janela, e, a cada desafio respondido, o Estudante recebe pontos e ganha níveis. Nenhuma informação técnica, aprovação ou configuração precisa ser feita pelo estudante. No AVA II a página customizada é acessível a partir da página inicial, e o serviços é visualizado através da mesma da mesma forma que no Moodle (Figura 9).

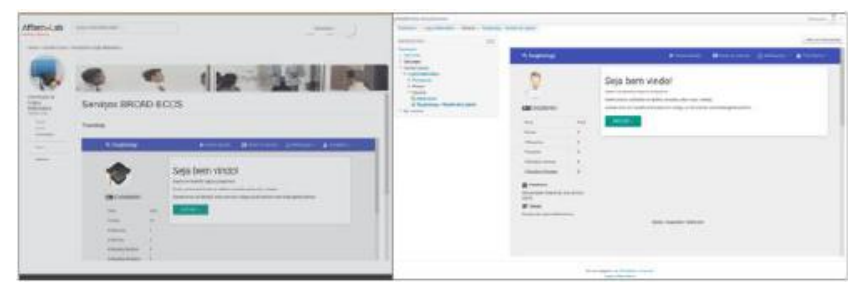

Figura 9 - Etapa III no Moodle (Esq.) e no AVA II (Dir.).

\subsection{Resultados}

A avaliação realizada demonstra a viabilidade da proposta, dos conceitos e das tecnologias envolvidas na integração de serviços educacionais ao AVA sob o ponto de vista de ECOS, cobrindo um ciclo completo de inclusão da infraestrutura necessária na forma de um plugin pelo Apoio/Desenvolvedor, a configuração de um curso por um Facilitador e o acesso por um Estudante sob o ponto de vista desses atores. Para cumprir as etapas planejadas, esse estudo de caso passa pelos componentes da proposta. Durante sua execução, foi possível observar que, do ponto de vista de atores não técnicos como Administrador, Professor e Estudante o uso da abordagem proposta para a integração e acesso ao serviço externo não exige configurações técnicas.

A execução em duas plataformas com características diferentes sob os pontos de vista arquitetural, técnico e gerencial evidencia a abrangência da arquitetura proposta, ao mesmo tempo em que manteve as características originais de cada AVA sob o ponto de vista dos atores. Em relação aos pontos de melhoria, foram observadas a necessidade de informações detalhadas dos escopos exigidos e a busca por serviços compatíveis a partir de uma loja ou canal de distribuição.

\section{CONSIDERAÇÕES FINAIS}

O trabalho apresentado propõe a aplicação da abordagem de ecossistemas como forma de favorecer o reuso e o compartilhamento de recursos e soluções. Para tanto utilizou-se uma plataforma tecnológica comum adaptada aos AVA atuais em um domínio caracterizado pela fragmentação e múltiplas implementações. A avaliação realizada apresenta indícios de que a proposta pode ajudar a resolver o problema observado no domínio de $e$-learning. Este fato foi evidenciado a partir de uma pesquisa exploratória com profissionais desse domínio tanto na área acadêmica quanto de treinamento.

Os AVAs são sistemas de informação em que as dificuldades no acompanhamento das inovações e necessidade de integração se destacam. No entanto, estas dificuldades não se restringem a esse tipo de sistema e o estudo de como ampliar a abordagem proposta para outros domínios é um trabalho futuro. Cabe assim expandir a avaliação para estudar sua aplicação tanto na academia quanto na área de treinamento. Adicionalmente, pretende-se avançar em relação às limitações observadas para auxiliar no entendimento dos escopos exigidos por determinado serviço. Por fim, é necessária a construção de um canal de distribuição para facilitar a busca por serviços compatíveis.

\section{REFERÊNCIAS}

[1] Experience API (xAPI). Acessado em 12 de outubro de 2015, de Advanced Distributed Learning http://www.adlnet.gov/tla/experience-api.

[2] Anrdt, J. E Dibbern, J. Co-Innovation in a Service Oriented Strategic Network. IEEE International Conference on Services Computing, 2006.SCC '06. , p. 285 - 288. 2006.

[3] Barbosa, O. Santos, R.; Alves, C.; Werner, C. e Jansen, S. Chapter 4: A systematic mapping study on software ecosystems from a three-dimensional perspective. Software Ecosystems: Analyzing and Managing Business Networks in the Software Industry. Edward Elgar Publishing, 2013.

[4] Berger, T., Pfeiffer, R. H., Tartler, R., Dienst, S., Czarnecki, K., Wąsowski, A. e She, S. Variability mechanisms in 
software ecosystems. Information and Software Technology, 56(11), 1520-1535. 2014.

[5] Bosch J. 2012. Software ecosystems: Taking software development beyond the boundaries of the organization. $J$. Syst. Softw. 85, 7 (July 2012), 1453-1454.

[6] Brodo, J. A. Today's Ecosystem of e-Learning. Trainer Talk, Professional Society for Sales \& Marketing Training, Vol. 3, No 4, 2006.

[7] Chang, V. e Gutl, C. E-Learning Ecosystem (ELES) - A Holistic Approach for the Development of more Effective Learning Environment for Small-to-Medium Sized Enterprises (SMEs), Proc. of IEEE Int. Digital EcoSystems Tech. Conf. (IEEE-DEST 2007), Cairns, Australia. 2007.

[8] Cowley, J., Chanley, S., Downes, S., Holstrom, L., Ressel, D., Siemens, G. \&Weisburgh, M. Preparing Students for Elearning, E-learnspace, 2002.

[9] Diniz, H., Silva E., Gama, K. Uma Arquitetura de Referência para Plataforma de Crowdsensing em Smart Cities. XI Brazilian Symposium on Information System, Goiânia, GO, May 26-29, 2015.

[10] Docebo: E-Learning Market Trends \& Forecast 2014-2016. Acesso em 07 de janeiro de 2015. Docebo: https://www.docebo.com/landing/learning-managementsystem/elearning-market-trends-and-forecast-2014-2016docebo-report.php, 2014.

[11] Dodero, J.M.; Palomo-Duarte, M.; Ruiz-Rube, I.; Traverso, I.; Mota, J.M.; Balderas, A., "Learning Technologies and Semantic Integration of Learning Resources," in IEEE Revista Iberoamericana de Tecnologias del Aprendizaje, vol.10, no.1, pp.11-16, Feb. 2015.

[12] Dong, B., Zheng, Q., Yang, J., Li, H. \&Qiao, M.An ELearning Ecosystem Based on Cloud Computing Infrastructure, Advanced Learning Technologies, 2009. ICALT 2009. Ninth IEEE Inter. Conf. on.pp.125, 127. 2009.

[13] Dowries, S. Models for Sustainable Open Educational Resources. Interdisciplinary Journal of Knowledge and Learning Objects, 3, 29-44. 2007

[14] Dresch, A.; Lacerda, D.; Antunes Jr, Design Science Research: A Method for Science and Technology Advancement. Springer International Publishing, 2015.

[15] Fragoso, O. G., Santaolaya, R., Munoz, S. J., Valenzuela, B. D., e Rojas, J. C. In Central America and Panama Convention Integration of learning Web services into learning management systems (CONCAPAN XXXIV). IEEE (pp. 1-6). 2014.

[16] Gutiérrez-Carreón, G. Daradoumis, T. e Jorba, J. Integrating Learning Services in the Cloud: An Approach that Benefits Both Systems and Learning. Educational Technology \& Society, 18(1), 145-157. 2015.

[17] Gutl, C. \& Chang, V. The use of Web 2.0 Technologies and Services to support E-Learning Ecosystem to develop more effective Learning Environments. ICDEM 2008, Tiruchirappalli, India. 2008.

[18] Hruska, M.; Medford., A; Murphy, J. (2015). Learning Ecosystems Using the Generalized Intelligent Framework for
Tutoring (GIFT) and the Experience API (xAPI). AIED 2015 Workshop Proceedings, Vol. 6. 2015.

[19] Jensen, S.; Finkelstein, A.; Brinkkemper, S.A sense of community: A research agenda for software ecosystems. In: 31st Inter. Conf. on Software Engineering - Companion Volume, 2009. ICSE-Companion 2009, pp. 187-190.

[20] Laanpere, M., Põldoja, H., \& Normak, P. Designing Dippler-a Next-Generation TEL System. In Open and Social Technologies for Networked Learning (pp. 91-100). Springer Berlin Heidelberg. 2013.

[21] Leong, Peter e Miao, Chunyan. Ubiquitous digital ELearning ecosystem. In: Digital Ecosystems and Technologies, 2008. DEST 2008. 2nd IEEE International Conferenceon. IEEE, 2008. p. 346-351.

[22] Lettner, D.; Angerer, F.; Prähofer, H. e Grünbacher, P.; A case studyon software ecosystemcharacteristics in industrial automation software. In Proceedings of the 2014 International Conference on Software and System Process (ICSSP 2014). ACM, New York, NY, USA, 40-49. 2014.

[23] Marinos, A.; Moschoyiannis, S.; KRAUSE, P. Towards a restful infrastructure for digital ecosystems. International Journal of Electronic Business, v. 9, n. 5-6, p. 484-498, 2011.

[24] Nars, M. \& Ouf, S. An Ecosystem in e-Learning using cloud computing as platform and Web2.0. The Research Bulletin of Jordan ACM,p. 134-140, NEA. 2011.

[25] Olinpo, G., Persico, D., Sarti, L., \&Tavella, M. On the concept of database of multimedia learning material. In: Proc. of World Conference on Computers and Education, Amsterdam, Australia, 1990, Northern Holland, pp 431-436.

[26] Petterson, O. Software ecosystems and e-Learning: recent developments and future prospects. MEDES '09: Int. ACM Conf. on Manag. of Emergent Digital EcoSystems, 2009.

[27] Ensino superior registra mais 7,3 milhões de estudantes. 2014. Acessado em 19 de dezembro de 2015. Portal Brasil: http://www.brasil.gov.br/educacao/2014/09/ensino-superiorregistra-mais-de-7-3-milhoes-de-estudantes.

[28] Serrano, M., Serrano, M. Cavalcante, C. Arquitetura de Software de Referência para Sistemas de Informação Governamentais. XI Brazilian Symposium on Information System, Goiânia, GO, May 26-29, 2015.

[29] Veiga, W.; Campos, F.; Braga, R.; David, J. M. LUDOS: uma Infraestrutura para Gamificação em Ecossistemas de Elearning. Anais do XXVI Simpósio Brasileiro de Informática na Educação (SBIE 2015). p. 459-469.

[30] Weller, M. Virtual learning environments: Using, choosing and developing your VLE. Routledge, 2007.

[31] Wholin, C.; Runeson, P.; Host, M.; Ohlsson, M. Regnell, B. e Wesslén, A.Experimentation in Software Engineering. Springer Berlin Heidelberg, 2012.

[32] Wilkinson, D. The Intersection of Learning Architecture and Instructional Design in e-Learning. e-Technologies in Engineering Education: Learning Outcomes Providing Future Possibilities. ECI Symposium Series, 2002. 